\title{
Pollen extract in association with vitamins provides early pain relief in patients affected by chronic prostatitis/chronic pelvic pain syndrome
}

\author{
TOMMASO CAI ${ }^{1}$, FLORIAN M.E. WAGENLEHNER ${ }^{2}$, LORENZO GIUSEPPE LUCIANI $^{1}$, DANIELE TISCIONE ${ }^{1}$, \\ GIANNI MALOSSINI ${ }^{1}$, PAOLO VERZE ${ }^{3}$, VINCENZO MIRONE ${ }^{3}$ and RICCARDO BARTOLETTI $^{4}$ \\ ${ }^{1}$ Department of Urology, Santa Chiara Regional Hospital, Trento, Italy; ${ }^{2}$ Clinic and Polyclinic for Urology, Child Urology \\ and Andrology, University Hospital of Giessen und Marburg, Justus-Liebig University, Giessen, Germany; \\ ${ }^{3}$ Department of Urology, University Federico II, Naples; ${ }^{4}$ Department of Urology, University of Florence, Florence, Italy
}

Received February 4, 2014; Accepted June 30, 2014

DOI: 10.3892/etm.2014.1861

\begin{abstract}
The therapeutic efficacy for chronic prostatitis/chronic pelvic pain syndrome (CP/CPPS) is currently unsatisfactory. The aim of the present study was to assess the safety and efficacy of pollen extract in association with vitamins (DEPROX 500 ${ }^{\circledR}$ ) in males with CP/CPPS. All patients with a diagnosis of $\mathrm{CP} / \mathrm{CPPS}$ attending the same urologic centre between March and October 2012 were enrolled in this randomised controlled phase III study. Participants were randomised to receive oral capsules of DEPROX $500^{\circledR}$ (two capsules every $24 \mathrm{~h}$ ) or ibuprofen $(600 \mathrm{mg}$, one tablet three times a day) for four weeks. The National Institutes of Health Chronic Prostatitis Symptom Index (NIH-CPSI), International Prostate Symptom Score and Quality of Well-Being (QoL) questionnaires were used. In the intention-to-treat analysis, 87 males (25 class IIIa and 62 class IIIb) with a mean age of $33.6 \pm 5.9$ years were randomly allocated to the DEPROX $500^{\circledR}$ $(n=41)$ or ibuprofen $(n=46)$ treatment groups. At the follow-up examination (following one month of treatment), in the DEPROX $500{ }^{\circledR}$ group, $31 / 41$ patients $(75.6 \%)$ reported an improvement in quality of life, defined as a reduction of the NIH-CPSI total score by $\geq 25 \%$, compared with $19 / 46$ (41.3\%) in the control group $(\mathrm{P}=0.002)$. The greater improvement in the DEPROX $500^{\circledR}$ group compared with the ibuprofen group was statistically significant (treatment difference in the NIH-CPSI pain domain, -2.14 \pm 0.51 , $\mathrm{P}<0.001$; QoL scores, $\mathrm{P}=0.002)$. All patients were negative at the Meares-Stamey test evaluation. Adverse events were less frequent in the DEPROX $500^{\circledR}$ group than in the ibuprofen group. The DEPROX $500^{\circledR}$ treatment
\end{abstract}

Correspondence to: Dr Tommaso Cai, Department of Urology, Santa Chiara Regional Hospital, 9 Largo Medaglie d'Oro 9-38123, Trento, Italy

E-mail: ktommy@libero.it

Key words: chronic pelvic pain syndrome, pollen extract, chronic prostatitis symptom index, quality of life, prostatitis syndrome significantly improved total symptoms, pain and quality of life compared with ibuprofen in patients with CP/CPPS, without severe side-effects.

\section{Introduction}

Chronic prostatitis (CP) has been described as one of the most common illnesses in males aged $<50$ years (1), and exhibits different clinical presentations (2). According to the classification of the National Institutes of Health (NIH) (3), class III $\mathrm{CP} /$ chronic pelvic pain syndrome (CP/CPPS) is the most frequent category (4), in which either genitourinary symptoms or pain are usually found and the impact on quality of life is considerable (5). The efficacies of current therapies for CP/CPPS are unsatisfactory (6). Phytotherapeutics are a noteworthy option due to their generally minimal side-effects; however, few have been subjected to scientific scrutiny and prospective controlled clinical trials $(7,8)$. In previous years, a number of studies have shown that pollen extract preparations are able to yield a durable and marked reduction of symptoms in young males with CP/CPPS, with an improvement in semen quality and a significant reduction in the NIH-Chronic Prostatitis Symptom Index (CPSI) score (9-11). Previously, Wagenlehner et al demonstrated that a standardized pollen extract significantly improved the total symptoms, pain and Quality of Well-Being (QoL) scores in patients with inflammatory $\mathrm{CP} / \mathrm{CPPS}$ without severe side-effects, highlighting the role of the anti-inflammatory activity of pollen extract (12). In the last year, Cai et al demonstrated that pollen extract in association with vitamins significantly improved the total symptoms, pain and QoL scores in patients with non-inflammatory CP/CPPS without severe side-effects in a phase II study (10). Furthermore, the association with vitamins is likely to improve the antioxidant activity of the pollen extract as well as the protective effect on nerves and also reduce the pain in patients with inflammatory or non-inflammatory CP/CPPS (10). The aim of the present study was to assess the safety and efficacy of pollen extract in association with vitamins in comparison with ibuprofen in order to improve the quality of life of patients affected by CP/CPPS by the relief of pain. 


\section{Materials and methods}

Study design. In order to assess the safety and efficacy of pollen extract in association with vitamins (DEPROX 500 ${ }^{\circledR}$ ) in males with CP/CPPS, all consecutive patients with a clinical and instrumental diagnosis of CP/CPPS (class IIIa or b), attending the same urologic centre (Santa Chiara Regional Hospital, Trento, Italy) between March and October 2012 were screened for this prospective randomised controlled phase III study. The design of the study was in accordance with the guidelines for clinical trials in CP/CPPS described by the NIH Chronic Prostatitis Collaborative Research Network (13). No placebo arm was included. The possible biases caused by the lack of placebo arm were considered in the results analysis. No placebo run-in period was considered necessary due to the fact that all enrolled patients were not blinded. The main outcome measure was the improvement of quality of life at the end of the whole study period, defined as the symptomatic improvement in the pain domain of the NIH-CPSI. Clinical failure was defined as the persistence of low quality of life following the treatment (failure to obtain a reduction of the NIH-CPSI total score by $\geq 25 \%$ ), or the suspension of therapy for significant reported adverse effects (12). In addition, spontaneously reported adverse events, or those noted by the investigator, were recorded during the whole study period. The study was conducted in line with Good Clinical Practice guidelines, with the ethical principles laid down in the latest version of the Declaration of Helsinki. Written informed consent was obtained from all patients prior to treatment. Furthermore, this study was conducted in line with the Consolidated Standards of Reporting Trials statement (The Ottawa Hospital Research Institute, Ottawa, ON, Canada).

Study schedule. On arrival at the center, all eligible individuals provided their written informed consent and underwent baseline questionnaires, urological examination and the Meares-Stamey test that was performed by the same urologist in accordance with the procedure described in the European Association of Urology (EAU) guidelines (14). All patients who met the inclusion criteria undertook oral administration of DEPROX 500 ${ }^{\circledR}$ (two capsules every $24 \mathrm{~h}$ ) or ibuprofen (600 mg, one tablet three times a day) for four weeks. Ibuprofen was selected in accordance with the results obtained by Lee et al (15). Proton-pump inhibitors (PPIs) were not routinely used due to the fact that all patients with gastrointestinal bleeding or a history of duodenal or gastric ulcers were excluded. Enrolled patients were not blinded to the preventative treatment. All patients were assigned to the two groups (DEPROX 500 ${ }^{\circledR}$ and ibuprofen) according to a 1:1 randomization (Fig. 1). All patients were contacted by telephone on day 14 of the therapy to ensure correct timing and dose of treatment. Each subject was scheduled for a follow-up examination at 30 days from starting therapy, with a urological and microbiological examination and questionnaire collection.

Inclusion and exclusion criteria. Inclusion criteria were the presence of symptoms of pelvic pain for at least three months during the six months before study entry, according to the EAU

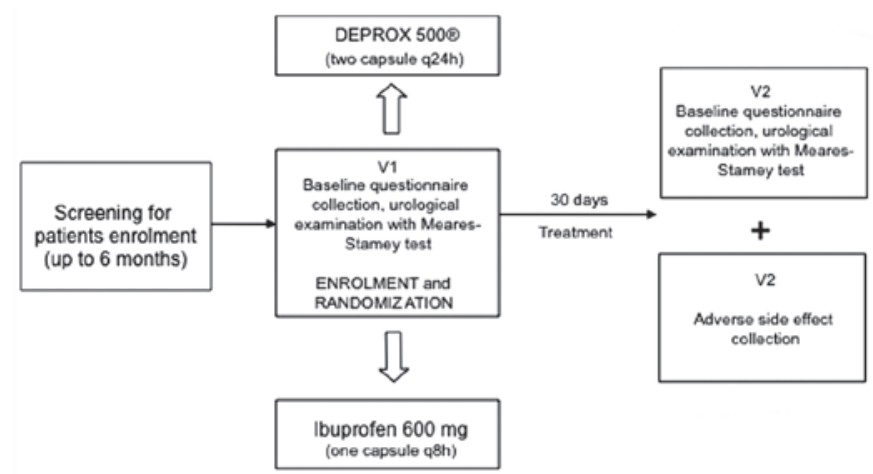

Figure 1. Study schedule. V1 = baseline, V2 = follow-up visit at 30 days q, every.

guidelines, a score in the pain domain of the NIH-CPSI (14) of $>7$ and a negative four-glass result in the Meares-Stamey test (12). Subjects $<18$ and $>65$ years of age, affected by major concomitant diseases, with known anatomical abnormalities of the urinary tract or with evidence of other urological diseases, and with residual urine volume $>50 \mathrm{ml}$ resulting from bladder outlet obstruction were excluded. Males with a reported allergy to pollen extract, who had recently ( $<4$ weeks) undergone oral or parental treatment or who were currently using prophylactic antibiotic drugs were also excluded. Additionally, all patients with a history of gastrointestinal bleeding or duodenal or gastric ulcers were excluded. All patients positive to tests for Chlamydia trachomatis $(\mathrm{Ct})$, Ureaplasma urealyticum, Neisseria gonorrhoeae, herpes viruses (HSV 1/2) and human papillomavirus (HPV) were also excluded.

Composition and characterization of the extracts used. All patients who were randomized to the DEPROX $500^{\circledR}$ group underwent oral administration of two tablets of DEPROX $500^{\circledR}$ in a single dose daily in the evening, in line with our previous study (10) and with the manufacturer's instructions (IDI Integratori Dietetici Italiani S.r.1, Sicily, Italy). Each administration contained $1 \mathrm{~g}$ pollen extract (500 $\mathrm{mg}$ per tablet), and vitamins B1, B2, B6, B9, B12 and PP. All compound analyses were carried out in accordance with the procedures described by Fiamegos et al (16). All patients randomized to the ibuprofen group received ibuprofen $(600 \mathrm{mg})$ three times per day.

Questionnaires and urological examinations. The validated Italian versions of the NIH-CPSI (17) and the International Prostate Symptom Score (IPSS) (18) questionnaires were administered to each patient. The questionnaires were self-administered when the patient arrived at the urologic centre. Furthermore, patient quality of life was measured by using an Italian version of the QoL scale, a validated, multi-attribute health scale (19). This scale was selected because it has been successfully applied to acute illnesses, whereas other quality of life scales, including the Short Form-36 (SF-36) Health Survey, are more suitable in chronic cases (20). Higher scores on the QoL scale reflect a higher quality of life. In accordance with the study by Nickel et al (21), prostatitis-like symptoms were considered significant at a pain 
score of $\geq 4$. The NIH-CPSI was also used in determining clinical therapy efficacy (21).

Sample collection and laboratory procedures. All samples were collected during the urological examination and immediately taken to the laboratory, under refrigerated conditions, analysed for cultures and aliquoted for DNA extraction and polymerase chain reaction for $\mathrm{Ct}$, Neisseria gonorrhoeae, HSV $1 / 2$ and HPV detection. All subjects included in the study underwent urinary culture for common bacteria, yeasts and urogenital mycoplasma. Microbiological culture was carried out in accordance with the methods described by Mazzoli et al (22). DNA extraction and purification from urine were performed using the EZ1 DNA Tissue kit (Qiagen SpA, Milan, Italy), as described in our previous study (22).

Statistical analysis. The primary target of the study was the symptomatic improvement in the pain domain of the NIH-CPSI. In order to analyse the homogeneity of the two groups, the baseline characteristics were compared using the Student's t-test and Mann-Whitney U test for continuous variables and by the $\chi^{2}$ test for categorical variables. The normal distribution of the variables was assessed using the Kolmogorov-Smirnov test. Data were analysed based on the intention-to-treat (ITT) approach. General characteristics of the study participants were expressed using descriptive statistics (means, standard deviations and ranges). The required sample size for the present study was calculated under the following conditions: Difference between the groups, $2 \pm 1$ score points in the NIH-CPSI pain domain; $\alpha$ error level, 0.05 two-sided; statistical power, $80 \%$; and anticipated effect size, Cohen's $d=0.5$. The calculation yielded $2 \times 39$ individuals per group. Randomization based on a single sequence of random assignments (simple randomization) was performed using a pseudo-random number generator software (Research Randomizer Version 4.0, Social Psychology Network, Wesleyan University, Middletown, CT, USA). Analysis of variance (ANOVA) was used for comparing the means. The Bonferroni adjustment test was also used at the second stage of the ANOVA. The effect size between the means (Cohen's d) was also calculated. The differences between the groups regarding the NIH-CPSI results were obtained using an ANOVA test. Statistical significance was achieved when $\mathrm{P}<0.05$. All reported $\mathrm{P}$-values were two-sided. Statistical analyses were performed using SPSS software, version 11.0 (SPSS, Inc., Chicago, IL, USA) for Apple-Macintosh.

\section{Results}

Patients. From the 115 patients attending the center for prostatitis-like symptoms during the study period, 94 were eventually enrolled and randomised. Out of the 21 patients excluded from the study, eight refused to be enrolled, six reported adverse effects to nonsteroidal anti-inflammatory drugs, two reported a clinical history of gastrointestinal ulcers and five elected to be treated in other centres. Additionally, seven patients were lost subsequent to randomisation and 87 males were finally enrolled (Fig. 2). The baseline questionnaire mean scores were $25.9 \pm 2.1,8.0 \pm 3.6$ and $0.55 \pm 0.15$ for NIH-CPSI, IPSS and QoL, respectively. Historical medical information and clinical data at enrolment are described in Table I. No statistically significant differences between the groups were identified.

Randomisation. Of the 87 enrolled patients (mean age 33.6 \pm 5.9 years), 41 received DEPROX 500 ${ }^{\circledR}$ (group A), and 46 received $600 \mathrm{mg}$ ibuprofen (group B). The treatment arms were comparable for all variables at the enrolment and randomisation visits.

Compliance with treatment schedule and adverse effects. In group A, $1 / 41$ patients $(2.4 \%)$ had mild adverse effects that did not require additional treatment (nausea), while in group B, $7 / 46$ patients $(15.2 \%)$ reported nausea and epigastric pain. In group A, 40 patients $(97.5 \%)$ were analysed subsequent to one being lost in follow-up. In group B, 38 patients $(82.6 \%)$ were analysed subsequent to two being lost to follow-up and four discontinuing therapy due to gastrointestinal adverse effects. The DEPROX $500^{\circledR}$ treatment was well tolerated in all the patients analysed, and no significant drug-related side-effects were identified. The analyses were carried out in the ITT (pollen extract, $n=41$; ibuprofen, $n=46$ ) and per protocol (PP) populations (pollen extract, $n=40$; ibuprofen, n=40) (Fig. 2).

Clinical and laboratory results at follow-up (after one month of treatment). At the follow-up examination, in the ITT set and DEPROX 500 ${ }^{\circledR}$ group, $31 / 41$ patients $(75.6 \%)$ reported an improvement of quality of life, defined as a reduction of the NIH-CPSI total score by $\geq 25 \%$, compared with $19 / 46(41.3 \%)$ in the control group $(\mathrm{P}=0.002)$. In the PP set and DEPROX 500 ${ }^{\circledR}$ group, $31 / 40$ patients $(77.5 \%)$ reported an improvement of quality of life, compared with $20 / 40(50.0 \%)$ in the control group $(\mathrm{P}=0.019)$. The questionnaire results at one month after treatment were as follows: NIH-CPSI, 12.8 \pm 2.20 ; IPSS, 7.6 \pm 1.58 ; and QoL, 0.69 \pm 0.10 in the DEPROX $500^{\circledR}$ group. By contrast the results in the control group were: NIH-CPSI, 19.5 \pm 2.10 ; IPSS, 8.00 \pm 2.81 ; and QoL, $0.59 \pm 0.18$. The higher improvement in the DEPROX $500{ }^{\circledR}$ group compared with the ibuprofen group was statistically significant (treatment difference in NIH-CPSI pain domain: ITT, $-2.14 \pm 0.51, \mathrm{P}<0.001$; PP, $-1.76 \pm 0.22, \mathrm{P}<0.001)$. Statistically significant differences were also reported in the NIH-CPSI $(\mathrm{P}<0.001)$, and QoL $(\mathrm{P}=0.002)$ scores between the two visits in the DEPROX $500^{\circledR}$ group and between the two groups (Figs. 3 and 4). No statistically significant differences were identified in the IPSS scores $(\mathrm{P}=0.43)$. All patients were negative at the Meares-Stamey test evaluation. All questionnaire results at the follow-up visit are presented in Table II. The results of the physical examinations, including vital signs, and laboratory examinations showed no relevant changes from the baseline.

Sub-analysis on the basis of CP/CPPS type. Of the 87 patients, $25(28.7 \%)$ showed inflammatory CP/CPPS (type IIIa), while $62(71.3 \%)$ exhibited type IIIb. A statistically significant difference was identified between the two groups in terms of pain relief and QoL improvement when stratified by CP/CPPS type. In fact, in the DEPROX $500^{\circledR}$ group, patients affected by type IIIb CP/CPPS showed higher QoL results and a lower pain level following treatment (the NIH-CPSI score was 
Table I. Baseline characteristics and clinical parameters at enrolment.

\begin{tabular}{|c|c|c|}
\hline Parameter & DEPROX $500^{\circledR}$ group & Ibuprofen group \\
\hline Patients, $\mathrm{n}$ & 41 & 46 \\
\hline Age, years $^{\mathrm{a}}$ & $33.8 \pm 6.78$ & $33.7 \pm 5.44$ \\
\hline \multicolumn{3}{|l|}{ Marital status, n (\%) } \\
\hline Married & $19(46.3)$ & $18(39.1)$ \\
\hline Unmarried & $22(53.7)$ & $28(60.8)$ \\
\hline \multicolumn{3}{|l|}{ Educational qualification, n (\%) } \\
\hline Primary school & $5(12.2)$ & $7(15.2)$ \\
\hline High school & $29(70.7)$ & $27(58.6)$ \\
\hline University & $7(17.1)$ & $12(26.2)$ \\
\hline \multicolumn{3}{|l|}{ Smoker status, n (\%) } \\
\hline Yes & $11(26.8)$ & $13(28.2)$ \\
\hline No & $30(73.2)$ & $33(71.8)$ \\
\hline Sexually active in the past month, $\mathrm{n}(\%)$ & $39(95.1)$ & $41(89.1)$ \\
\hline \multicolumn{3}{|l|}{ Sexual behaviour, n (\%) } \\
\hline 1 partner & $33(80.4)$ & $37(80.4)$ \\
\hline$>1$ partners & $8(19.6)$ & $9(19.6)$ \\
\hline \multicolumn{3}{|l|}{ Contraceptive use, n (\%) } \\
\hline Condom & $29(70.3)$ & $34(73.9)$ \\
\hline Coitus interruptus & $12(29.7)$ & $12(26.1)$ \\
\hline Start of CP history (months) ${ }^{\mathrm{a}}$ & $18.7 \pm 4.28$ & $19.1 \pm 3.99$ \\
\hline \multicolumn{3}{|l|}{ Symptoms score at baseline ${ }^{a}$} \\
\hline NIH-CPSI & $24.9 \pm 2.1$ & $25.5 \pm 3.0$ \\
\hline IPSS & $8.3 \pm 3.6$ & $8.0 \pm 2.5$ \\
\hline QoL & $0.57 \pm 0.17$ & $0.55 \pm 0.15$ \\
\hline \multicolumn{3}{|l|}{ Clinical presentation, n (\%) } \\
\hline Dysuria & $12(29.2)$ & $14(30.4)$ \\
\hline Urgency & $4(9.7)$ & $5(10.8)$ \\
\hline Dysuria + frequency & $6(14.6)$ & $5(10.8)$ \\
\hline Burning & $7(17.0)$ & $9(19.5)$ \\
\hline \multicolumn{3}{|l|}{ Pain, n (\%) } \\
\hline Perineal & $19(46.4)$ & $21(45.6)$ \\
\hline Scrotal & $4(9.7)$ & $4(8.7)$ \\
\hline Suprapubic & $8(19.6)$ & $9(19.6)$ \\
\hline Lower abdominal & $10(24.3)$ & $12(26.1)$ \\
\hline \multicolumn{3}{|l|}{ Pain frequency, n (\%) } \\
\hline Daily & $33(80.4)$ & $36(78.2)$ \\
\hline Weekly & $8(19.6)$ & $10(21.8)$ \\
\hline \multicolumn{3}{|l|}{ Sexual Symptoms, n (\%) } \\
\hline ED & $12(29.2)$ & $13(28.2)$ \\
\hline $\mathrm{PE}$ & $7(17.0)$ & $6(13.0)$ \\
\hline $\mathrm{ED}+\mathrm{EP}$ & $4(9.7)$ & $4(8.6)$ \\
\hline \multicolumn{3}{|l|}{ CP/CPPS type, n (\%) } \\
\hline Type a & $12(29.2)$ & $13(28.2)$ \\
\hline Type b & $29(70.8)$ & $33(71.8)$ \\
\hline
\end{tabular}

${ }^{a}$ Data are presented as the mean \pm standard deviation. CP, chronic prostatitis; NIH-CPSI, National Institutes of Health-Chronic Prostatitis Symptom Index; IPSS, International Prostate Symptom Score; QoL, Quality of Well-Being; CPPS, chronic pelvic pain syndrome; ED, erectile dysfunction; PE, premature ejaculation. 


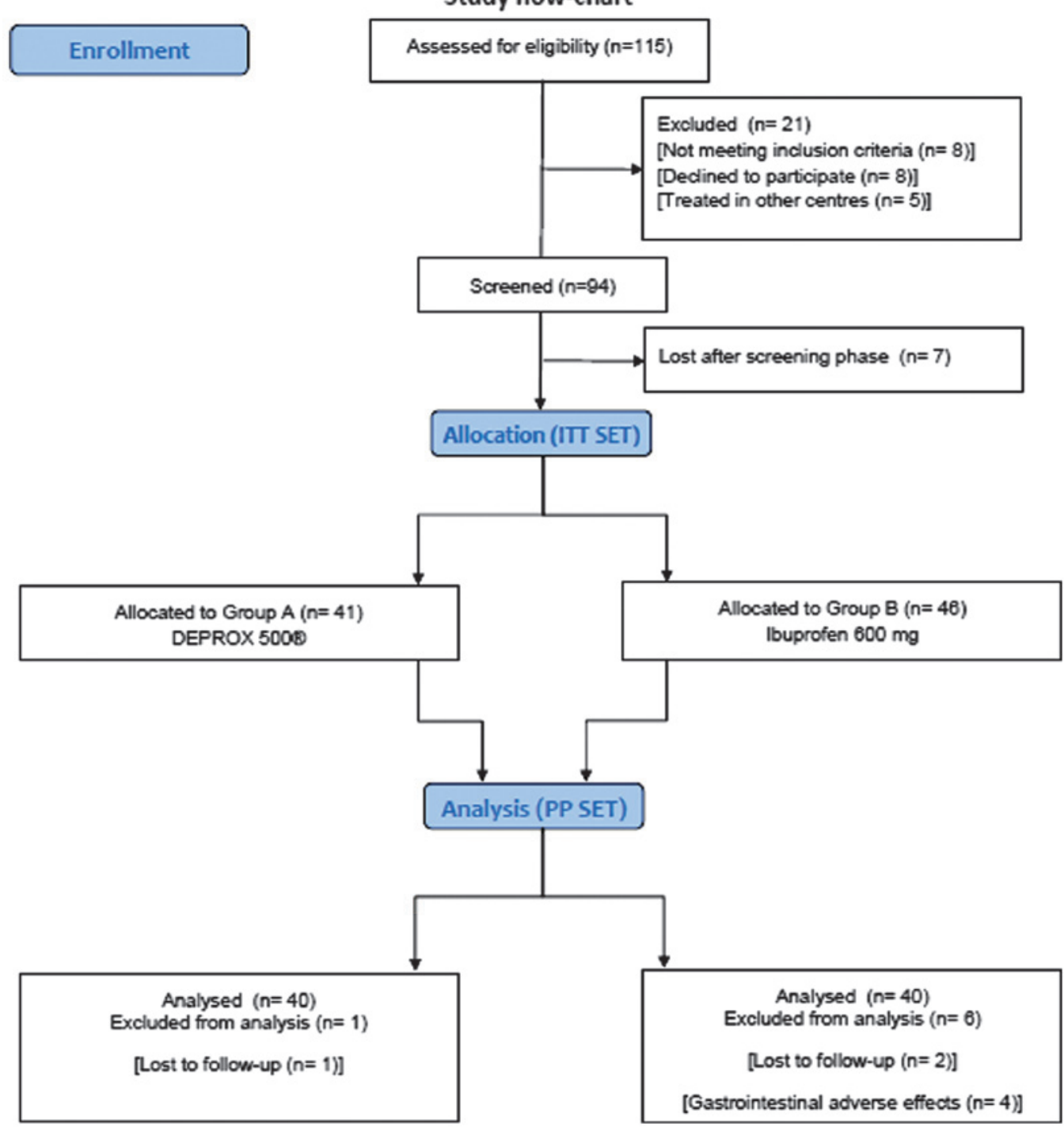

Figure 2. Study flow-chart according to the Consolidated Standards of Reporting Trials statement. ITT, intention-to-treat; PP, per protocol analysis.

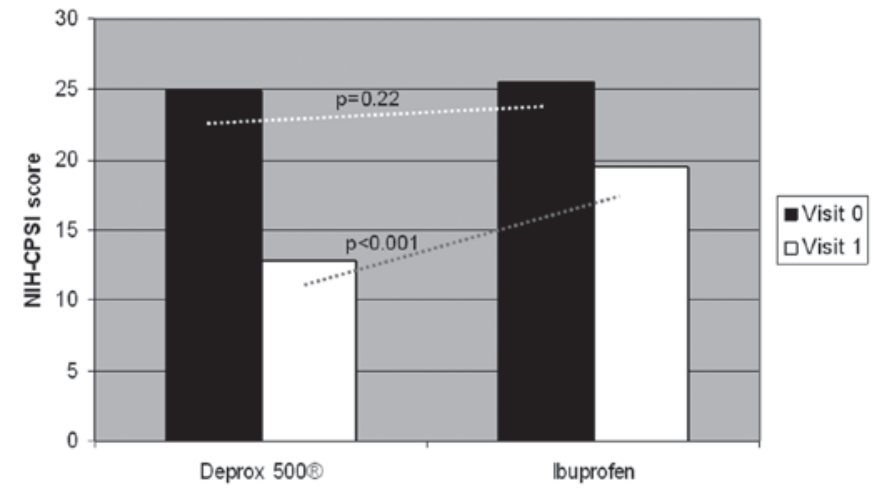

Figure 3. Statistically significant differences between the two visits in terms of the NIH-CPSI scores $(\mathrm{P}<0.001)$ between the two groups. NIH-CPSI, National Institutes of Health-Chronic Prostatitis Symptom Index.

$24.8 \pm 1.8$ at the enrolment versus $11.7 \pm 1.7$ at the follow-up visit; $\mathrm{P}<0.001)$ when compared with type IIIa CP/CPPS patients (Table III). No differences were reported between the ITT or PP sets.

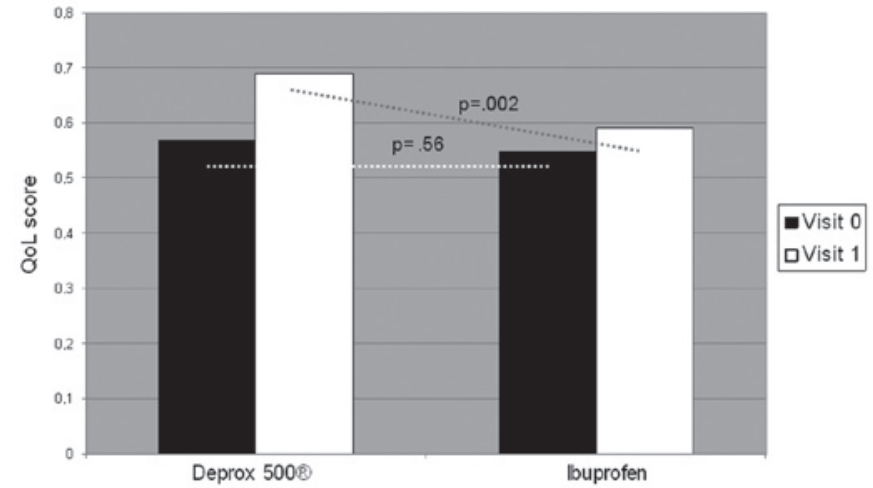

Figure 4. Statistically significant differences between the two visits in terms of the $\mathrm{QoL}$ scores $(\mathrm{P}=0.002)$ between the two groups. QoL, Quality of Well-Being.

\section{Discussion}

The major finding of the present study was that DEPROX $500^{\circledR}$ is able to provide early pain relief and improve the quality of 
Table II. Questionnaire results at the follow-up visit.

\begin{tabular}{|c|c|c|}
\hline Variable & DEPROX $500^{\circledR}$ group & Ibuprofen group \\
\hline NIH-CPSI $(\mathrm{P}<0.001)^{\mathrm{a}}$ & $12.8 \pm 2.20$ & $19.5 \pm 2.10$ \\
\hline IPSS $(\mathrm{P}=0.87)^{\mathrm{a}}$ & $7.6 \pm 1.58$ & $8.00 \pm 2.81$ \\
\hline $\mathrm{QoL}(\mathrm{P}=0.002)^{\mathrm{a}}$ & $0.69 \pm 0.10$ & $0.59 \pm 0.18$ \\
\hline Reduction in NIH-CPSI pain domain ${ }^{\mathrm{b}}, \mathrm{n}(\%)$ & $31(77.5)$ & $19(47.5)$ \\
\hline Efficacy outcomes (NIH-CPSI pain domain) $)^{\mathrm{a}, \mathrm{c}}$ & $-4.36 \pm 0.51$ & $-2.22 \pm 0.53$ \\
\hline Efficacy outcomes (NIH-CPSI pain domain) $)^{\mathrm{a}, \mathrm{d}}$ & $-3.86 \pm 0.21$ & $-2.10 \pm 0.20$ \\
\hline
\end{tabular}

${ }^{a}$ Data are presented as the mean \pm standard deviation; ${ }^{b}$ pain, $\mathrm{P}<0.003$; ' intention-to-treat analysis, treatment difference $-2.41 \pm 0.51, \mathrm{P}<0.001$; ${ }^{\mathrm{d}}$ per protocol analysis, treatment difference $-1.76 \pm 0.22, \mathrm{P}<0.001$. NIH-CPSI, National Institutes of Health-Chronic Prostatitis Symptom Index; IPSS, International Prostate Symptom Score; QoL, Quality of Well-Being.

Table III. Results of the sub-analysis on the basis of CP/CPPS type a or b.

\begin{tabular}{lcc}
\hline Variable & DEPROX 500 ${ }^{\circledR}$ group & Ibuprofen group \\
\hline Patients, n & 40 & 40 \\
Type a, n (\%) & $14(35)$ & $11(27.5)$ \\
Type b, n (\%) & $29(65)$ & $32(72.5)$ \\
NIH-CPSI $^{\mathrm{a}}$ & & \\
Type a & $13.1 \pm 1.8$ & $20.2 \pm 1.9$ \\
Type b & $11.7 \pm 1.7$ & $19.1 \pm 2.7$ \\
IPSS $^{\mathrm{a}}$ & & $7.9 \pm 3.1$ \\
Type a & $7.9 \pm 0.9$ & $8.0 \pm 2.2$ \\
Type b & $7.4 \pm 1.5$ & \\
QoL & & \\
Type a & & $0.57 \pm 0.2$ \\
Type b & $0.61 \pm 0.3$ & $0.60 \pm 0.1$ \\
\hline
\end{tabular}

a Data are presented as the mean \pm standard deviation. NIH-CPSI, National Institutes of Health-Chronic Prostatitis Symptom Index; IPSS, International Prostate Symptom Score; QoL, Quality of Well-Being; CP/CPPS, chronic prostatitis/chronic pelvic pain syndrome.

life in patients with $\mathrm{CP} / \mathrm{CPPS}$ without severe side-effects, when compared with ibuprofen. Furthermore, it was revealed that patients affected by type IIIb CP/CPPS may obtain greater advantages from this therapy. These findings lead to several points of discussion. Firstly, the early pain relief. In 2006, Elist (23), using a double-blind study with random distribution versus placebo, demonstrated the superiority of pollen extract versus placebo in terms of improvement in pain score and the filling and emptying symptoms from the start to the end of the treatment after six months of therapy. Additionally, in 2009, Wagenlehner et al (12) showed that pollen extract improved symptoms, pain and quality of life after 12 weeks of treatment in patients with this condition, with differences in favour of pollen extract at six weeks of treatment compared with the placebo, and the treatment being well tolerated. These two studies treated the patients for at least six weeks $(12,23)$. Consistent with our previous study (10), 30 days of treatment with DEPROX $5000^{\circledR}$ in the present study was able to provide significant results in terms of pain reduction when compared with ibuprofen. This effect is possibly due to the association between the pollen extract and vitamins B6 and B12 that improve the antioxidant activity of pollen extract with the protective effect on nerves. B vitamins including thiamine (B1), pyridoxine (B6) and cyanocobalamin (B12) are capable of antinociception in experimental animals with acute and chronic pain evoked by electrical, chemical and thermal stimulation, primary neuronal injury and diabetes $(24,25)$. Notably, several studies have demonstrated that certain B vitamins, particularly B6 and $\mathrm{B} 12$, are able to protect neurons from certain injuries $(26,27)$. The B vitamins, B1, B6 and B12, are clinically useful in the treatment of certain painful conditions including lumbago, sciatica, trigeminal neuralgia and chronic pain associated with diabetic polyneuropathy (28). Finally, we hypothesized that the early improvement on pain relief is due to the protective effect on nerves, and the following improvement in quality of life could be due to the antioxidant activity of pollen extract. Indeed, previous studies in which only pollen extract was administered demonstrated an improvement in quality of life and pain relief after $\geq 6$ weeks of treatment. Furthermore, on the basis of the sub-analysis, the patients that best obtained the important advantages from this therapy were those with non-inflammatory CP/CPPS. Contrasting with previous studies, the present study revealed that patients with non-inflammatory CP/CPPS showed improved results compared with those with inflammatory $\mathrm{CP} / \mathrm{CPPS}$. This is possibly due to the fact that the protective effect on nerves of $B$ vitamins occurs earlier than the anti-inflammatory effect of pollen extract. In this sense, DEPROX $5000^{\circledR}$ is able to provide improved results in terms of early pain reduction in patients with non-inflammatory CP/CPPS. DEPROX $500^{\circledR}$ was generally well tolerated over the full study period.

The present study had a few limitations that should be taken into account: The small number of enrolled patients, a short follow-up period, a selected patient population, the lack of control group and that this was not a blinded study. Given the lack of prove efficacy of conventional therapies, alternative treatment options are urgently required and pollen extract in association with vitamins should be an noteworthy option due to its generally low side-effects and promising results in terms of quality of life improvement. 
In conclusion, given the aforementioned limitations, DEPROX $500^{\circledast}$ significantly improved the total symptoms, pain, and quality of life compared with ibuprofen in patients with CP/CPPS, without severe side-effects.

\section{Acknowledgements}

The authors would like to thank Professor John Denton (Department of Modern Philology, University of Florence, Florence, Italy) for language revision of the study.

\section{References}

1. Collins MM, Stafford RS, O'Leary MP and Barry MJ: How common is prostatitis? A national survey of physician visits. J Urol 159: 1224-1228, 1998

2. Barbalias GA: Clinical and therapeutical guidelines for chronic prostatitis. from bacteriological importance to neuromuscular considerations. Eur Urol 37: 116-117, 2000.

3. Workshop Committee of the National Institute of Diabetes and Digestive and Kidney Disease (NIDDK). Chronic Prostatitis Workshop, Bethesda, MD, 7-8 December, 1995.

4. Schaeffer AJ: Classification (traditional and National Institutes of Health) and demographics of prostatitis. Urology 60 (6 Suppl): $5-7,2002$

5. Nickel JC: Role of alpha1-blockers in chronic prostatitis syndromes. BJU Int 101 (Suppl 3): 11-16, 2008.

6. Tuğcu V, Taşçi AI, Fazlioğlu A, et al: A placebo-controlled comparison of the efficiency of triple- and monotherapy in category III B chronic pelvic pain syndrome (CPPS). Eur Urol 51: 1113-1118, 2007.

7. Herati AS and Moldwin RM: Alternative therapies in the management of chronic prostatitis/chronic pelvic pain syndrome. World J Urol 31: 761-766, 2013.

8. Shoskes DA, Zeitlin SI, Shahed A and Rajfer J: Quercetin in men with category III chronic prostatitis: a preliminary prospective, double-blind, placebo-controlled trial. Urology 54: 960-963, 1999.

9. Rugendorff EW, Weidner W, Ebeling L and Buck AC: Results of treatment with pollen extract (Cernilton $\mathrm{N}$ ) in chronic prostatitis and prostatodynia. Br J Urol 71: 433-438, 1993.

10. Cai T, Luciani LG, Caola I, et al: Effects of pollen extract in association with vitamins (DEPROX $500^{\circledR}$ ) for pain relief in patients affected by chronic prostatitis/chronic pelvic pain syndrome: results from a pilot study. Urologia 80 (Suppl 22): 5-10,2013.

11. Kamijo T, Sato S and Kitamura T: Effect of cernitin pollen-extract on experimental nonbacterial prostatitis in rats. Prostate 49 : 122-131, 2001.

12. Wagenlehner FM, Schneider H, Ludwig M, Schnitker J, Brähler E and Weidner W: A pollen extract (Cernilton) in patients with inflammatory chronic prostatitis-chronic pelvic pain syndrome: a multicentre, randomised, prospective, double-blind, placebo-controlled phase 3 study. Eur Urol 56: 544-551, 2009.

13. Propert KJ, Alexander RB, Nickel JC, et al; Chronic Prostatitis Collaborative Research Network: Design of a multicenter randomized clinical trial for chronic prostatitis/chronic pelvic pain syndrome. Urology 59: 870-876, 2002.
14. Grabe M, Bjerklund-Johansen TE, Botto H, et al: Guidelines on Urological Infections. European Association of Urology, Arnhem, The Netherlands, p66, 2012.

15. Lee CB, Ha US, Lee SJ, Kim SW and Cho YH: Preliminary experience with a terpene mixture versus ibuprofen for treatment of category III chronic prostatitis/chronic pelvic pain syndrome. World J Urol 24: 55-60, 2006.

16. Fiamegos YC, Nanos CG, Vervoort J and Stalikas CD: Analytical procedure for the in-vial derivatization-extraction of phenolic acids and flavonoids in methanolic and aqueous plant extracts followed by gas chromatography with mass-selective detection. J Chromatogr A 1041: 11-18, 2004.

17. Giubilei G, Mondaini N, Crisci A, et al: The Italian version of the National Institutes of Health Chronic Prostatitis Symptom Index. Eur Urol 47: 805-811, 2005.

18. Badía X, García-Losa M and Dal-Ré R: Ten-language translation and harmonization of the International Prostate Symptom Score: developing a methodology for multinational clinical trials. Eur Urol 31: 129-140, 1997.

19. Kaplan RM, Bush JW and Berry CC: Health status: types of validity and the index of well-being. Health Serv Res 11: 478-507, 1976.

20. Ernst EJ, Ernst ME, Hoehns JD and Bergus GR: Women's quality of life is decreased by acute cystitis and antibiotic adverse effects associated with treatment. Health Qual Life Outcomes 3: 45, 2005.

21. Nickel JC, Downey J, Hunter D and Clark J: Prevalence of prostatitis-like symptoms in a population based study using the National Institutes of Health chronic prostatitis symptom index. J Urol 165: 842-845, 2001.

22. Mazzoli S, Cai T, Rupealta V, et al: Interleukin 8 and anti-chlamydia trachomatis mucosal IgA as urogenital immunologic markers in patients with $C$. trachomatis prostatic infection. Eur Urol 51: 1385-1393, 2007.

23. Elist J: Effects of pollen extract preparation Prostat/Poltit on lower urinary tract symptoms in patients with chronic nonbacterial prostatitis/chronic pelvic pain syndrome: a randomized, double-blind, placebo-controlled study. Urology 67: 60-63, 2006.

24. Jolivalt CG, Mizisin LM, Nelson A, et al: B vitamins alleviate indices of neuropathic pain in diabetic rats. Eur J Pharmacol 612: 41-47, 2009.

25. Yu CZ, Liu YP, Liu S, Yan M, Hu SJ and Song XJ: Systematic administration of B vitamins attenuates neuropathic hyperalgesia and reduces spinal neuron injury following temporary spinal cord ischaemia in rats. Eur J Pain 18: 76-85, 2014.

26. Wang ZB, Gan Q, Rupert RL, Zeng YM and Song XJ. Thiamine, pyridoxine, cyanocobalamin and their combination inhibit thermal, but not mechanical hyperalgesia in rats with primary sensory neuron injury. Pain 114: 266-277, 2005.

27. Hung KL, Wang CC, Huang CY and Wang SJ: Cyanocobalamin, vitamin B12, depresses glutamate release through inhibition of voltage-dependent $\mathrm{Ca}^{2+}$ influx in rat cerebrocortical nerve terminals (synaptosomes). Eur J Pharmacol 602: 230-237, 2009.

28. Mäder R, Deutsch H, Siebert GK, et al: Vitamin status of inpatients with chronic cephalgia and dysfunction pain syndrome and effects of a vitamin supplementation. Int J Vitam Nutr Res 58: 436-441, 1988 